\title{
Editorial
}

\section{Concrete and Structure with Recycled Aggregate}

\author{
Alex Li, ${ }^{1}$ François Buyle-Bodin, ${ }^{2}$ Yves Delmas, ${ }^{1}$ Dimitar Dontchev, ${ }^{3}$ and Christophe Petit ${ }^{4}$ \\ ${ }^{1}$ Laboratory of Civil Engineering, Université de Reims Champagne-Ardenne, 51687 Reims, France \\ ${ }^{2}$ UFR of Geography and Management, Université de Lille 1 Sciences et Techniques de Lille, 59100 Lille, France \\ ${ }^{3}$ University of Chemical Technology and Metallurgy Sofia, 1756 Sofia, Bulgaria \\ ${ }^{4}$ Department of Civil Engineering, Université de Limoges, 19300 Egletons, France \\ Correspondence should be addressed to Alex Li; alex.li@univ-reims.fr
}

Received 1 December 2013; Accepted 1 December 2013

Copyright (C) 2013 Alex Li et al. This is an open access article distributed under the Creative Commons Attribution License, which permits unrestricted use, distribution, and reproduction in any medium, provided the original work is properly cited.

In recent years, importance and necessity of using the recycled aggregate are recognized because of the depletion of quality primary aggregates and greater awareness of environmental protection. It is now widely accepted that there is a significant potential for reclaiming and recycling demolished debris for use in value added applications to maximize economic and environmental benefits. There is increasing demand and interest in aggregates from nontraditional sources such as from industrial byproducts and recycled construction and demolition wastes. The purpose of this special issue is to summarize the various researches on concrete and structure with recycled aggregate. A few papers invited in this special issue give their contribution to advance research in this field.

The first paper of this special issue presents the cellular concrete bricks obtained by using a lightweight mortar with recycled expanded polystyrene aggregate instead of sandy materials. This type of concrete bricks is lighter and more flexible, which makes it less vulnerable to cracking walls due to soil displacements. The developed bricks have good mechanical properties and flexural strength and less permeable.

The second paper deals with the cementing potential of pulverized oyster shell, rich in calcium, when mixed with fly ash and soil. Authors indicate that more the pulverized oyster shell adds, lower the strength will get both in the soil and lime specimen. The soil specimens containing fly ash gradually gain strength as curing proceeds.

The third paper shows that the impact properties of the recycled aggregate concrete exhibit strong strain-rate dependency and increase the increase of peak strain-rate.
The transition point from low strain-rate sensitivity to high sensitivity decreases with the increase of matrix strength. Authors indicate that the energy absorption capacity of recycled aggregate concretes specimens is lower than that of natural aggregate concretes.

The fourth paper presents an optimization of the mixing proportion of recycled aggregate concrete by using neural networks based on genetic algorithms. This method will contribute to improve the usage of the recycled aggregate in the construction industry and to reduce the waste in the construction process.

In the fifth paper, the effects of ground waste concrete powder coming from the attached paste/mortar on water demand for normal consistency, setting time, fluidity, and compressive strength of cement were analyzed.

The last paper presents the fire resistance and the relevant thermal properties of high-performance concrete (HPC) with fly ash. The effect of temperature from 200 to $500^{\circ} \mathrm{C}$ on compressive strength, cubic compressive strength, cleavage strength, flexural strength, and the ultrasonic velocity of the high-performance concrete with fly ash was studied.

$$
\begin{array}{r}
\text { Alex Li } \\
\text { François Buyle-Bodin } \\
\text { Yves Delmas } \\
\text { Dimitar Dontchev } \\
\text { Christophe Petit }
\end{array}
$$



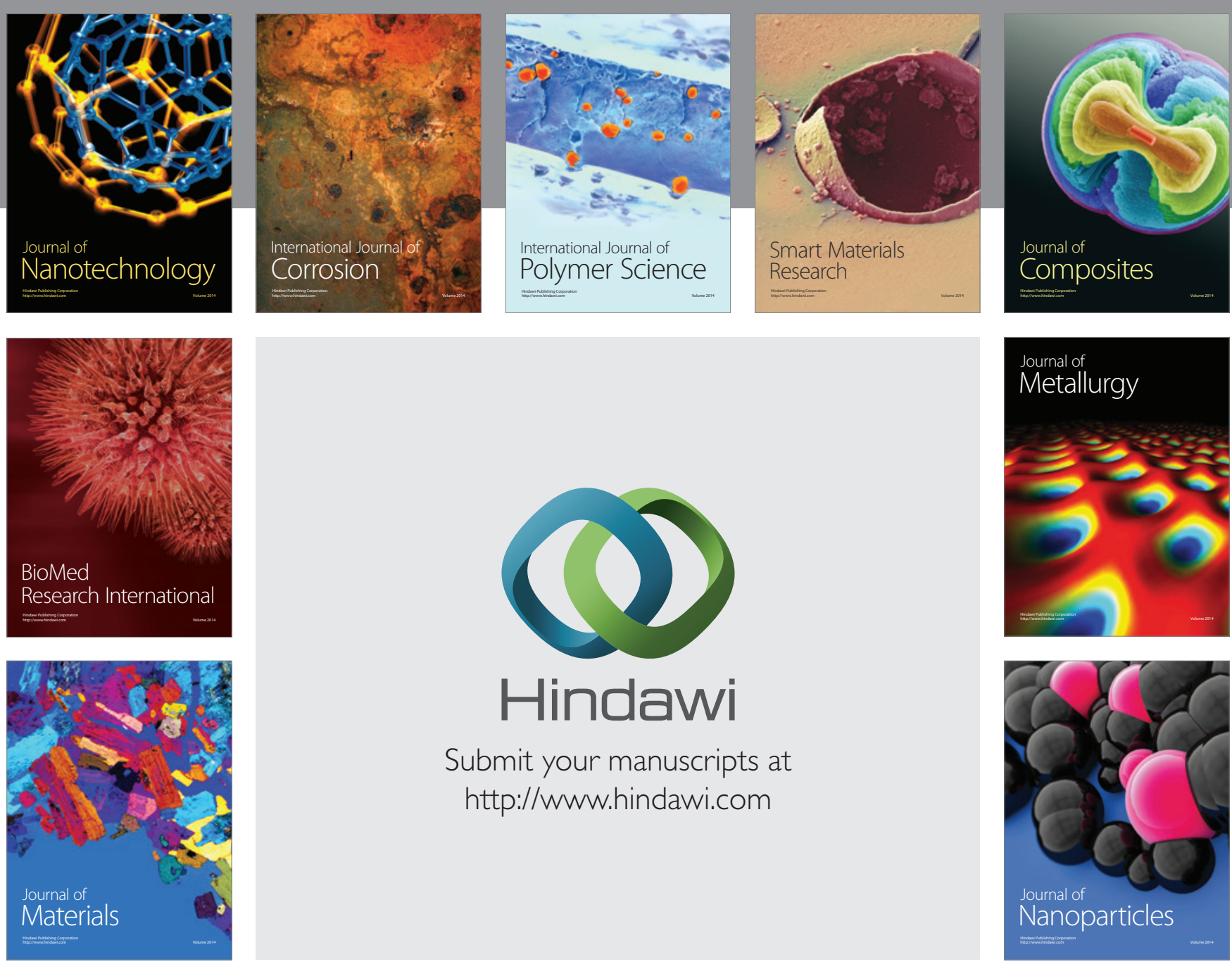

Submit your manuscripts at http://www.hindawi.com
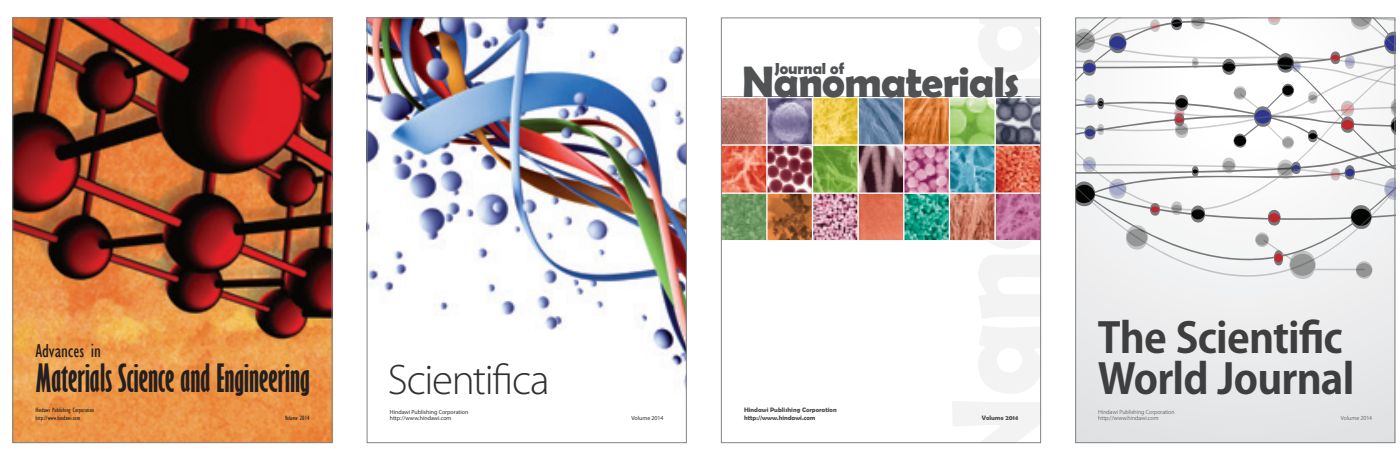

\section{The Scientific World Journal}
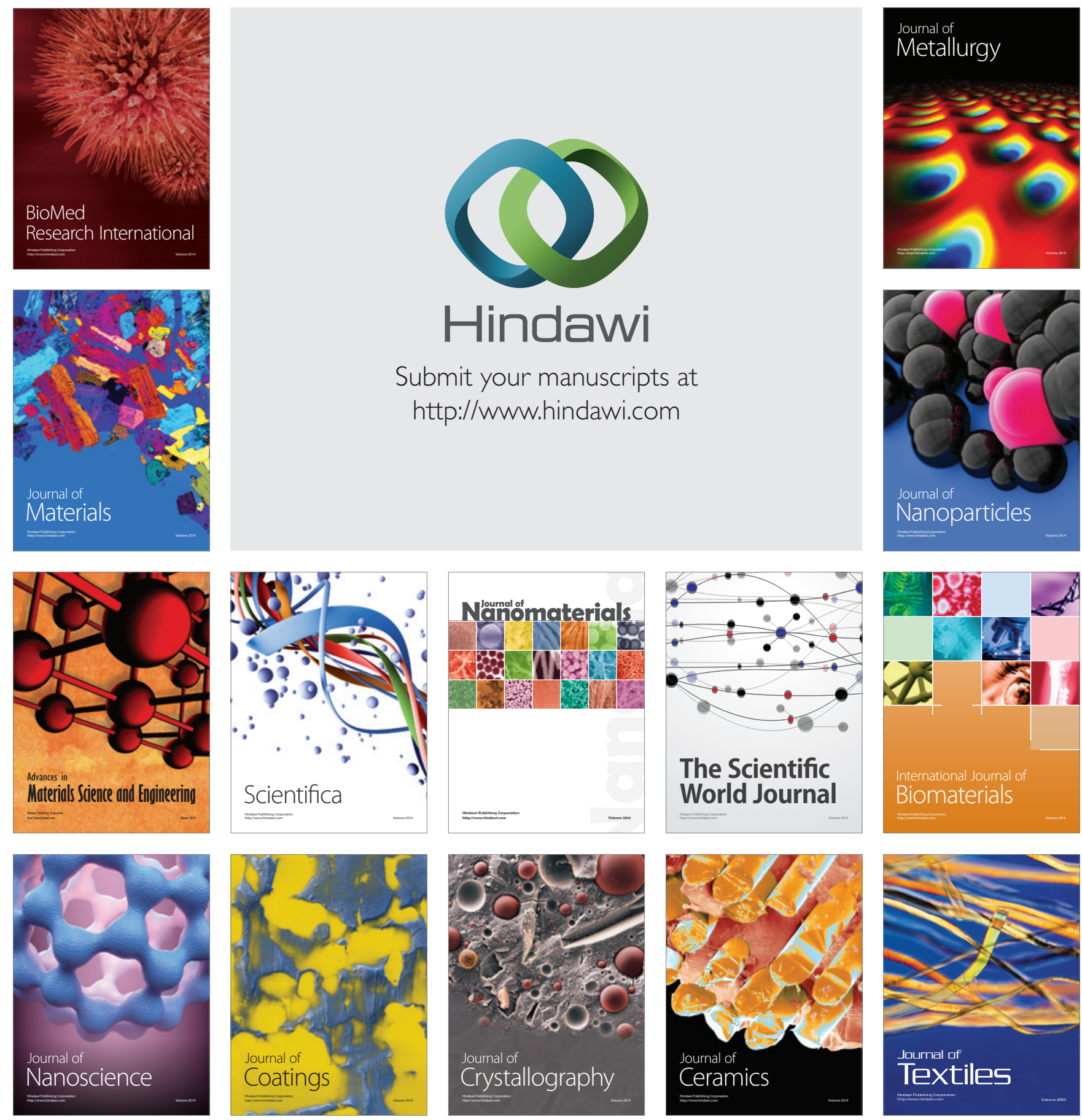